\title{
Discovery and biological evaluation of a novel highly potent selective butyrylcholinsterase inhibitor
}

Qi Li ${ }^{\text {a }}$, Shuaishuai Xing a, Ying Chen ${ }^{\mathrm{c}}$, Qinghong Liao ${ }^{\mathrm{c}}$, Baichen Xiong ${ }^{\mathrm{a}}$, Siyu He ${ }^{\mathrm{d}}$, Weixuan Lu a, Yang Liu a , Hongyu Yang a , Qihang Li a , Feng Feng c, e, Wenyuan Liu a, Yao Chen ${ }^{\text {b, }}{ }^{*}$, Haopeng Sun ${ }^{\text {a, }}$ *

${ }^{a}$ School of Pharmacy, China Pharmaceutical University, Nanjing, 211198, People's Republic of China

${ }^{b}$ School of Pharmacy, Nanjing University of Chinese Medicine, Nanjing, 210023, People's Republic of China

c Department of Natural Medicinal Chemistry, China Pharmaceutical University, Nanjing, 211198, People's Republic of China

$d$ State Key Laboratory of Natural Medicines, Jiangsu Key Laboratory of Carcinogenesis and Intervention, School of Basic Medicine and Clinical Pharmacy, China Pharmaceutical University, Nanjing 210009, People's Republic of China e Jiangsu Food and Pharmaceutical Science College, No.4 Meicheng Road, Huai'an, 223003, People's Republic of China

1. Table S1. Shape-based model for screening, Chemdiv ID, structure, Lipinski's values and CDOCKER interaction energy.

2. Figure S1. (A) ee $\mathrm{AChE}$ and (B) eq $\mathrm{BChE}$ inhibitory rate of 13 compounds under 10 $\mu \mathrm{M}$.

3. Figure S2. Dose-response curves and $\mathrm{IC}_{50}$ determination. Concentration-dependent inhibition of eqBChE by (A) V028-5347, (B) V009-8861, (C) V013-8206, (D) 8012-9656 and (E) tacrine as positive control. Concentration-dependent inhibition of $h \mathrm{BChE}$ by $\mathbf{8 0 1 2 - 9 6 5 6}$ is presented in F.

4. Figure S3. Binding modes of 8012-9656 with $h \mathrm{BChE}$ (PDB code: $5 \mathrm{k} 5 \mathrm{e}$ ). 80129656 is shown in blue, only important residues of the receptor are presented in yellow. Hydrophobic interactions are shown in pink and hydrogen bonds are shown in green.

5. Figure S4. Alignment of docking mode of 8012-9656 with original ligand donepezil in the receptor. The pocket surface is presented in blue; donepezil is shown in yellow and 8012-9656 is shown in green.

6. Figure S5. Everyday body weights of mice in control and 8012-9656 groups.

7. Figure S6. Standard curve of mouse $A \beta_{1-42}$ Elisa kit.

8. Chemistry

8.1 ${ }^{1} \mathrm{H}$ NMR and ${ }^{13} \mathrm{C}$ NMR of intermediate compounds $\mathbf{2}, \mathbf{3}$ and $\mathbf{5}$.

8.2 HPLC, ${ }^{1} \mathrm{H}$ NMR, ${ }^{13} \mathrm{C}$ NMR and HRMS of compound 8012-9656.

8.3 HPLC, ${ }^{1} \mathrm{H}$ NMR, ${ }^{13} \mathrm{C}$ NMR and HRMS of synthesized positive control, tacrine.

9. Table S2. Molecular Formula Strings of all compounds. 
Table S1. Shape-based model for screening, Chemdiv ID, structure, Lipinski's values and CDOCKER interaction energy.

\begin{tabular}{|c|c|c|c|c|c|c|c|}
\hline \multirow{2}{*}{$\begin{array}{l}\text { Shape-based } \\
\text { model in Fig. } \\
2 \mathrm{~A} \text { and/or } 2 \mathrm{~B}\end{array}$} & \multirow[b]{2}{*}{ Chemdiv ID } & \multirow[b]{2}{*}{ Structure } & \multicolumn{4}{|c|}{ Lipinski's values } & \multirow{2}{*}{$\begin{array}{c}\text {-CDOCKER } \\
\text { interaction } \\
\text { energy } \\
(\mathrm{kcal} / \mathrm{mol})\end{array}$} \\
\hline & & & $\begin{array}{c}\text { MW } \\
(\mathrm{g} / \mathrm{mol})\end{array}$ & HD & HA & $\log D$ & \\
\hline $2 \mathrm{~A}$ & $8015-2476$ & & 460.49 & 2 & 4 & 6.48 & 47.45 \\
\hline $2 \mathrm{~A}$ and $2 \mathrm{~B}$ & 3434-1301 & & 417.46 & 1 & 3 & 5.90 & 43.86 \\
\hline $2 \mathrm{~B}$ & V028-5347 & & 425.49 & 0 & 6 & 4.37 & 48.88 \\
\hline $2 \mathrm{~A}$ & V011-7286 & & 509.41 & 0 & 6 & 4.46 & 47.50 \\
\hline $2 \mathrm{~B}$ & K630-0106 & & 420.51 & 2 & 3 & 6.52 & 52.21 \\
\hline $2 \mathrm{~A}$ and $2 \mathrm{~B}$ & D101-0057 & & 435.48 & 0 & 7 & 3.41 & 49.26 \\
\hline $2 \mathrm{~A}$ and $2 \mathrm{~B}$ & M769-0012 & & 402.50 & 1 & 4 & 4.67 & 48.61 \\
\hline
\end{tabular}




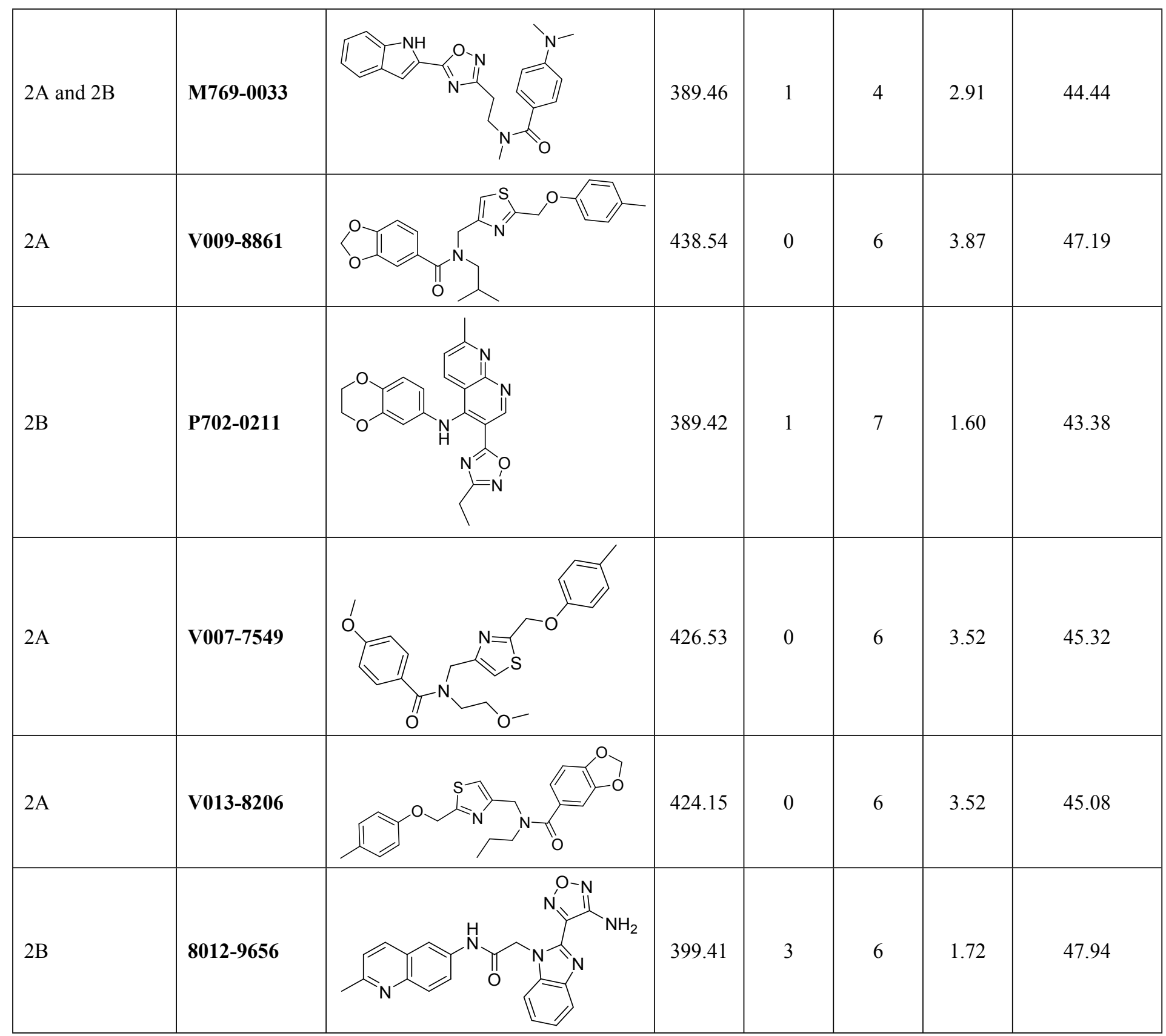

Note: full name of four screened hit compounds shown in Figure 3.

(1-(3',4'-dimethoxy-[1,1'-biphenyl]-3-yl)-1H-imidazol-5-yl)(isoindolin-2-

yl)methanone (V028-5347)

$N$-isobutyl-N-((2-((p-tolyloxy)methyl)thiazol-4-yl)methyl)benzo[d][1,3]dioxole-5carboxamide (V009-8861)

$N$-propyl-N-((2-((p-tolyloxy)methyl) thiazol-4-yl)methyl)benzo[d][1,3]dioxole-5carboxamide (V013-8206)

2-(2-(4-amino-1,2,5-oxadiazol-3-yl)-1H-benzo[d]imidazol-1-yl)-N-(2-methylquinolin6-yl)acetamide (8012-9656) 
Figure S1. (A) eeAChE and (B) eqBChE inhibitory rate of 13 compounds under 10 $\mu \mathrm{M}$.

A AChE inhibitory rate of compounds under $10 \mu \mathrm{M}$

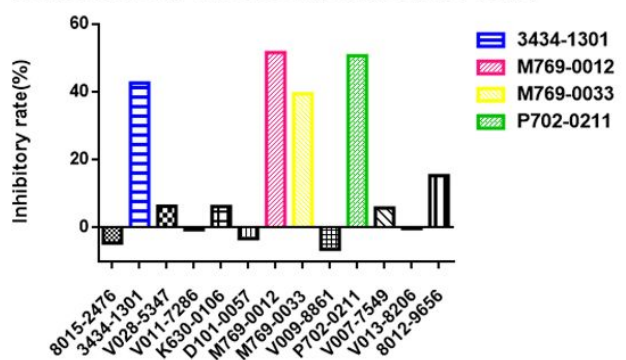

B BuChE inhibitory rate of compounds under $10 \mu \mathrm{M}$

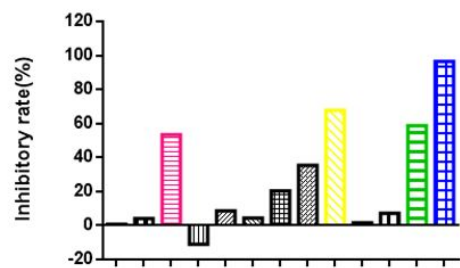

的 $06-1001$

S06-1002

므 $\mathrm{s} 06-1003$

[I.⿴囗十 $506-1004$ 
Figure S2. Dose-response curves and $\mathrm{IC}_{50}$ determination. Concentration-dependent inhibition of $e q \mathrm{BChE}$ by (A) V028-5347, (B) V009-8861, (C) V013-8206, (D) 80129656 and (E) tacrine as positive control. Concentration-dependent inhibition of $h \mathrm{BChE}$ by 8012-9656 is presented in F.
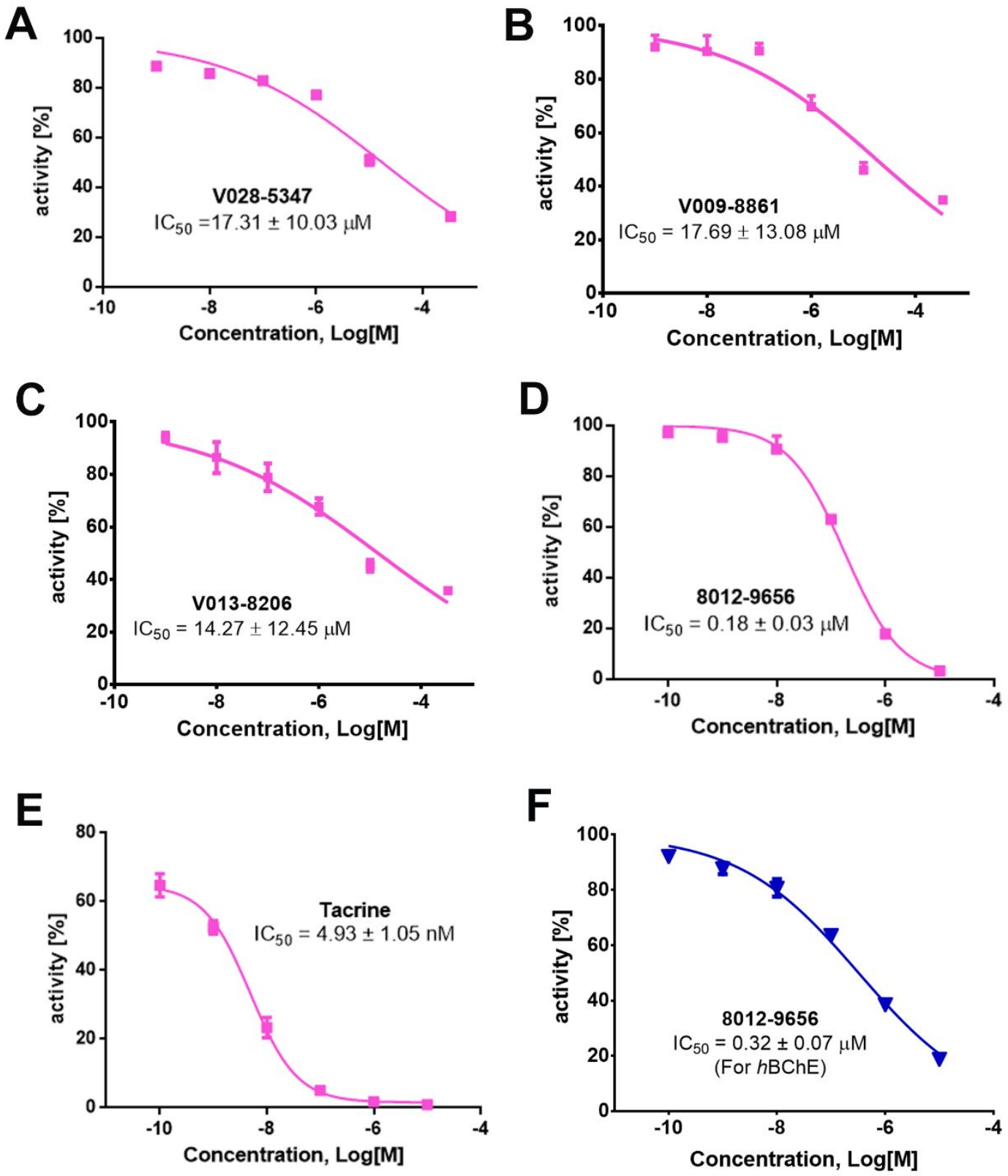
Figure S3. Binding modes of 8012-9656 with $h \mathrm{BChE}$ (PDB code: $5 \mathrm{k} 5 \mathrm{e}$ ). 8012-9656 is shown in blue, only important residues of the receptor are presented in yellow. Hydrophobic interactions are shown in pink and hydrogen bonds are shown in green.

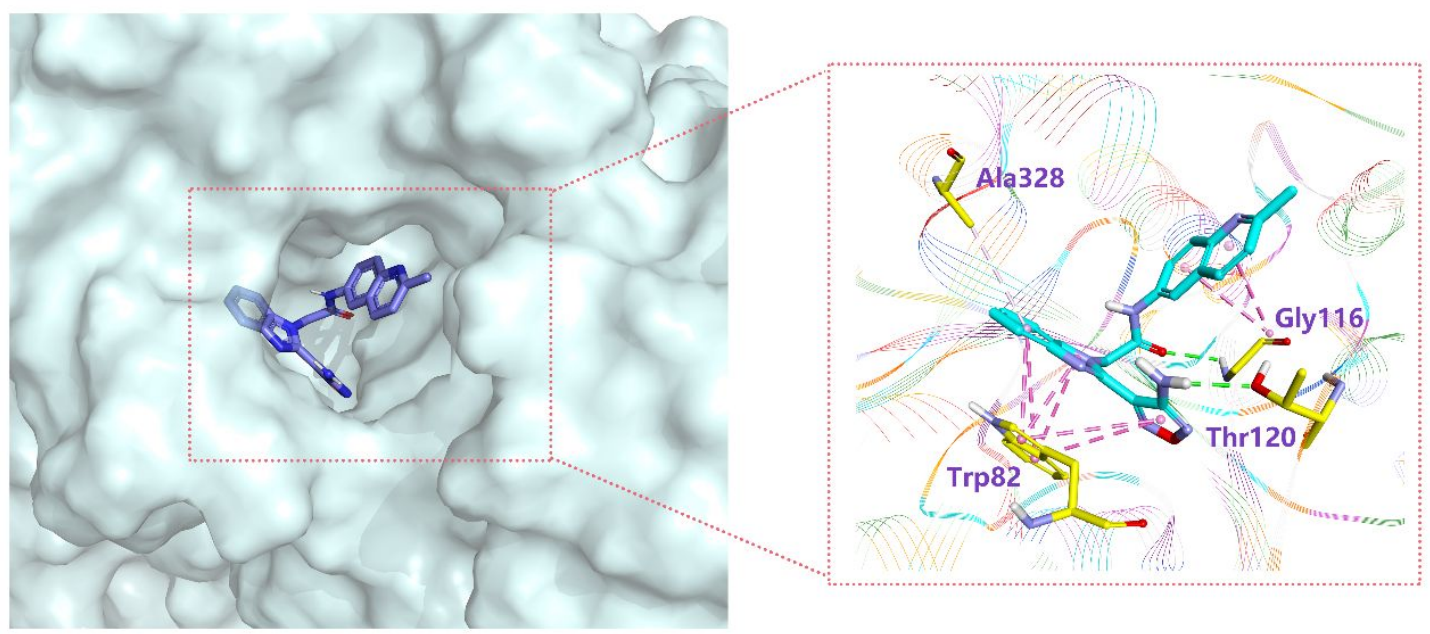


Figure S4. Alignment of docking mode of 8012-9656 with original ligand donepezil in the receptor. The pocket surface is presented in blue; donepezil is shown in yellow and 8012-9656 is shown in green.

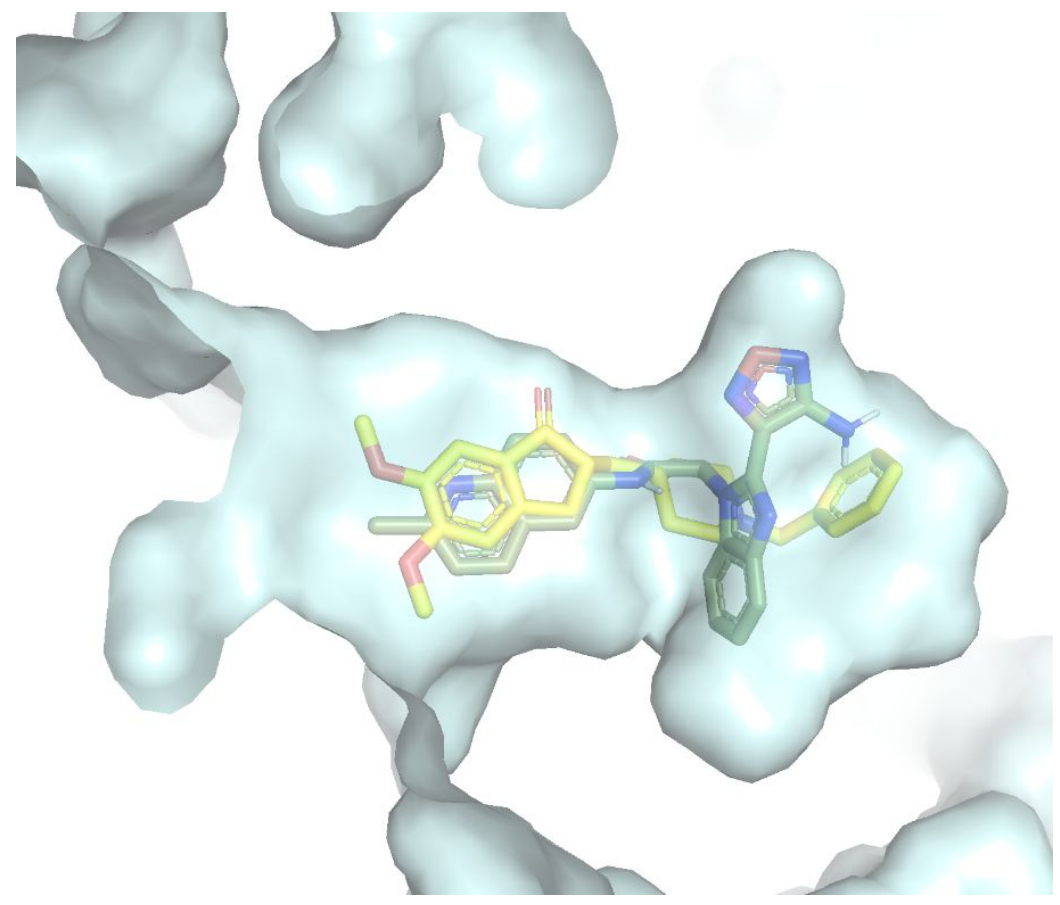


Figure S5. Everyday body weights of mice in control and 8012-9656 groups.

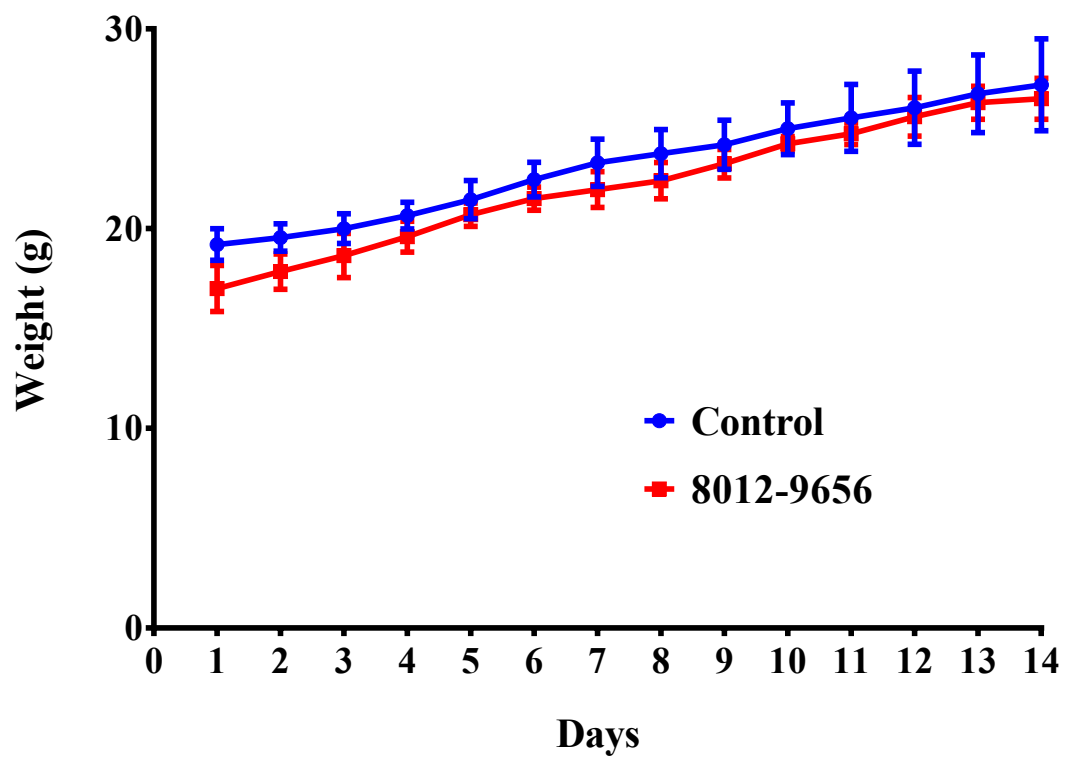


Figure S6. Standard curve of mouse $A \beta_{1-42}$ Elisa kit.

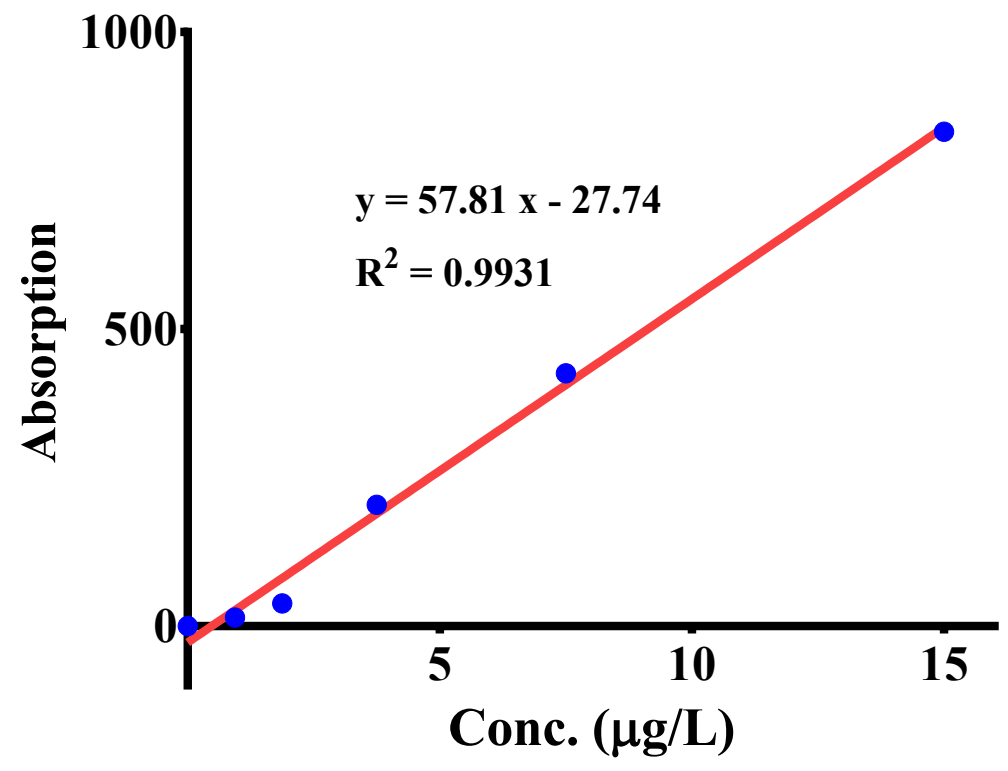


${ }^{1} \mathrm{H}$ NMR of intermediate compound 2.

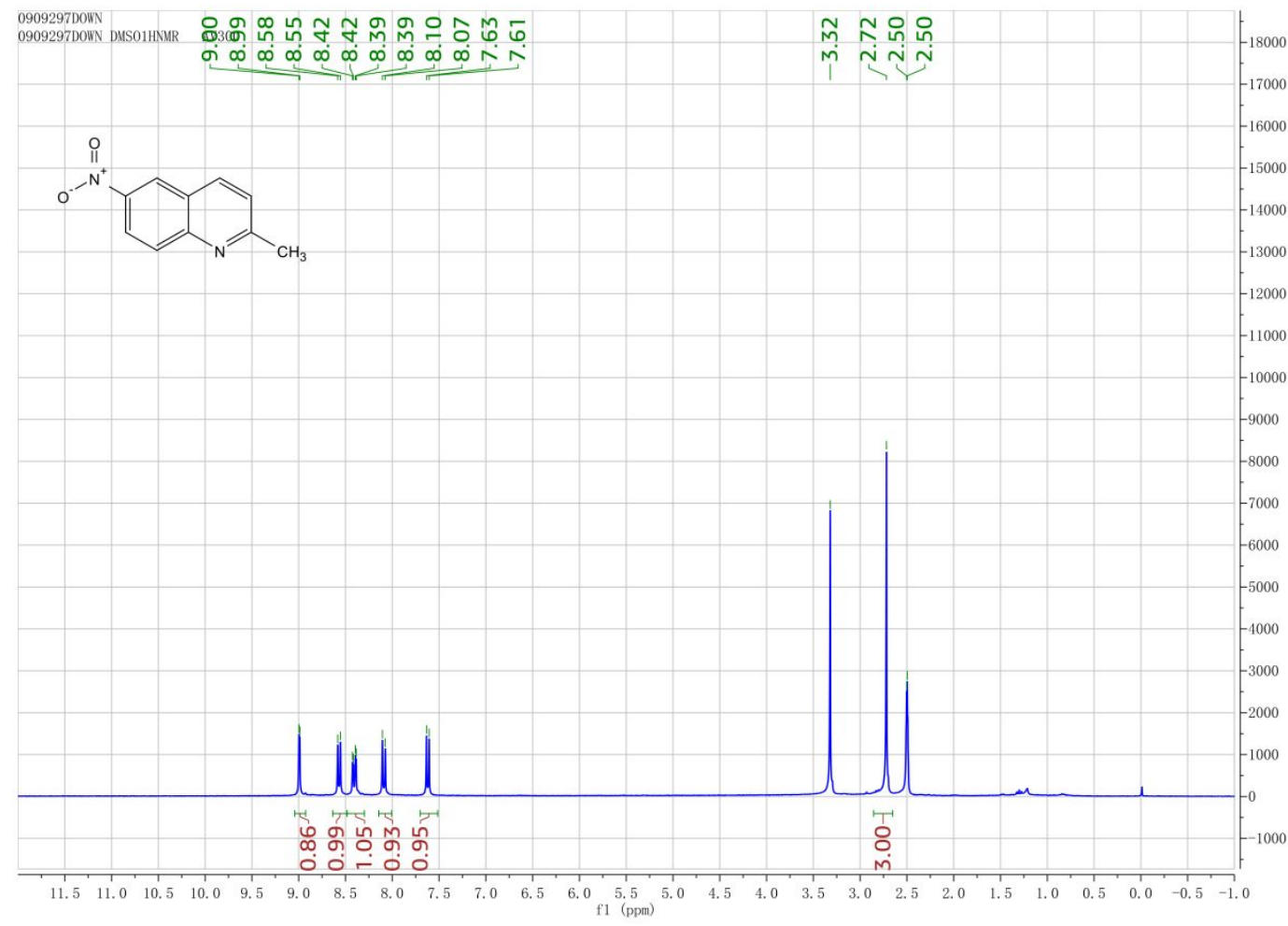

${ }^{13} \mathrm{C}$ NMR of intermediate compound 2.

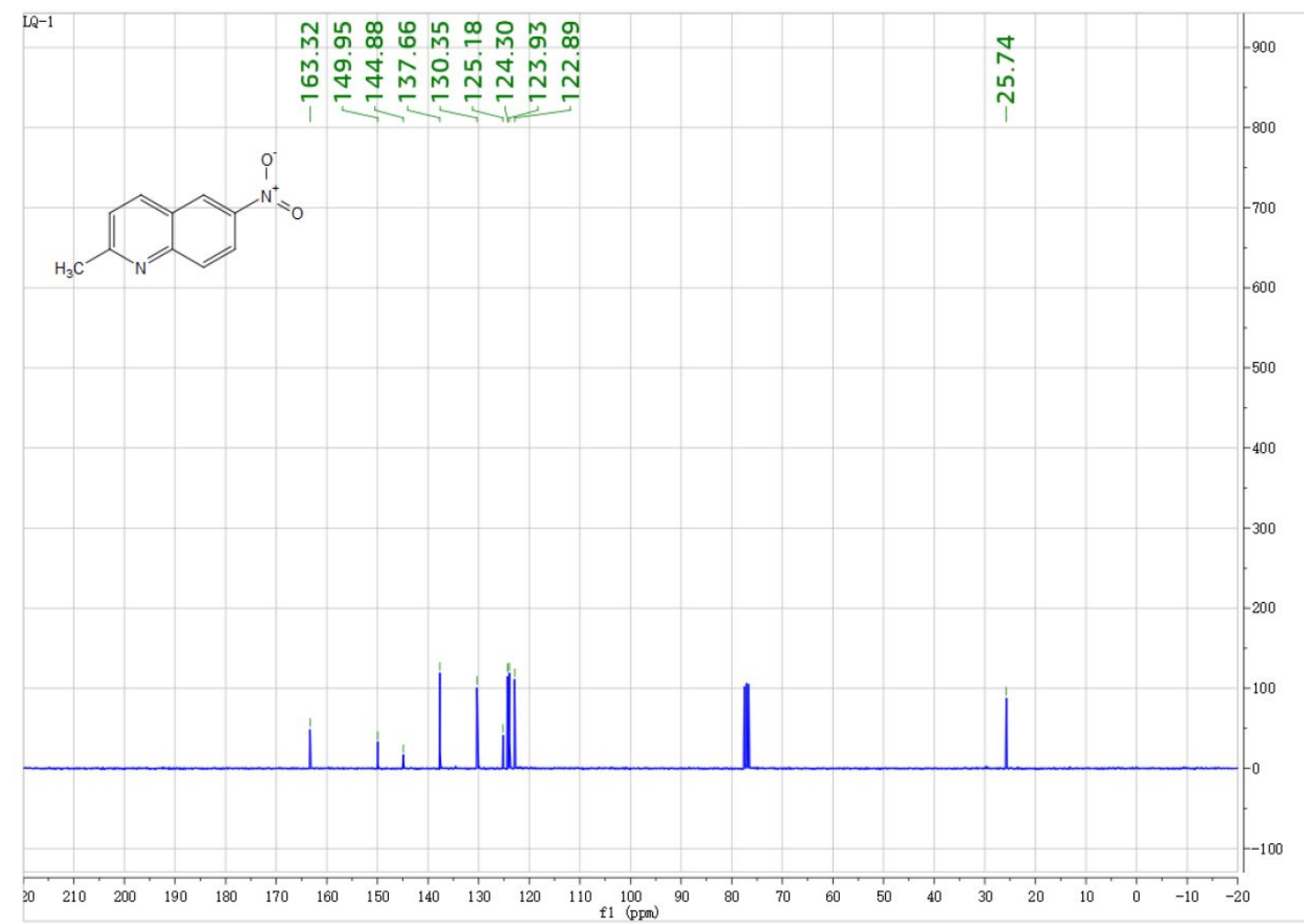

${ }^{1} \mathrm{H}$ NMR of intermediate compound 3. 


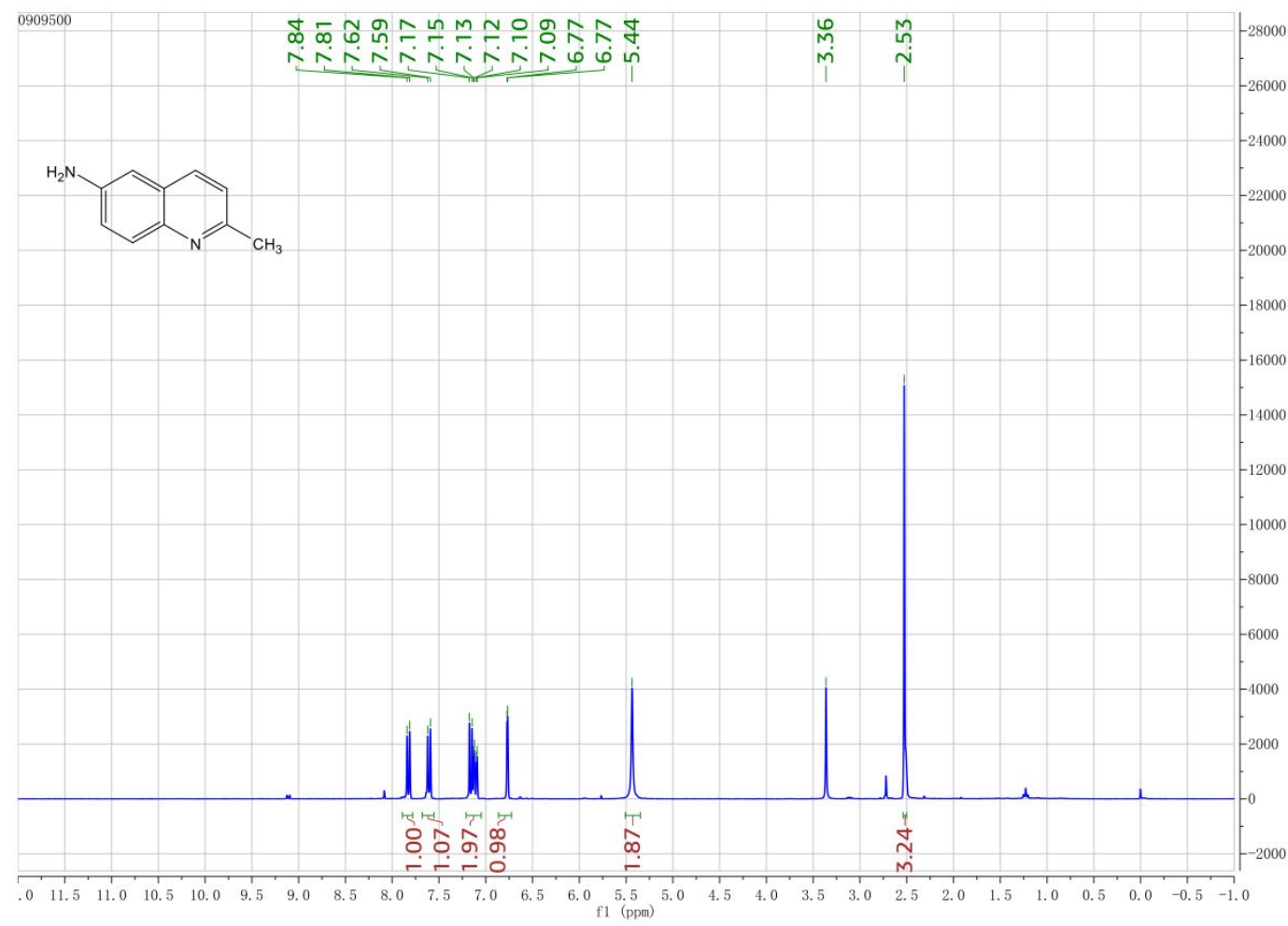

${ }^{13} \mathrm{C}$ NMR of intermediate compound 3 .

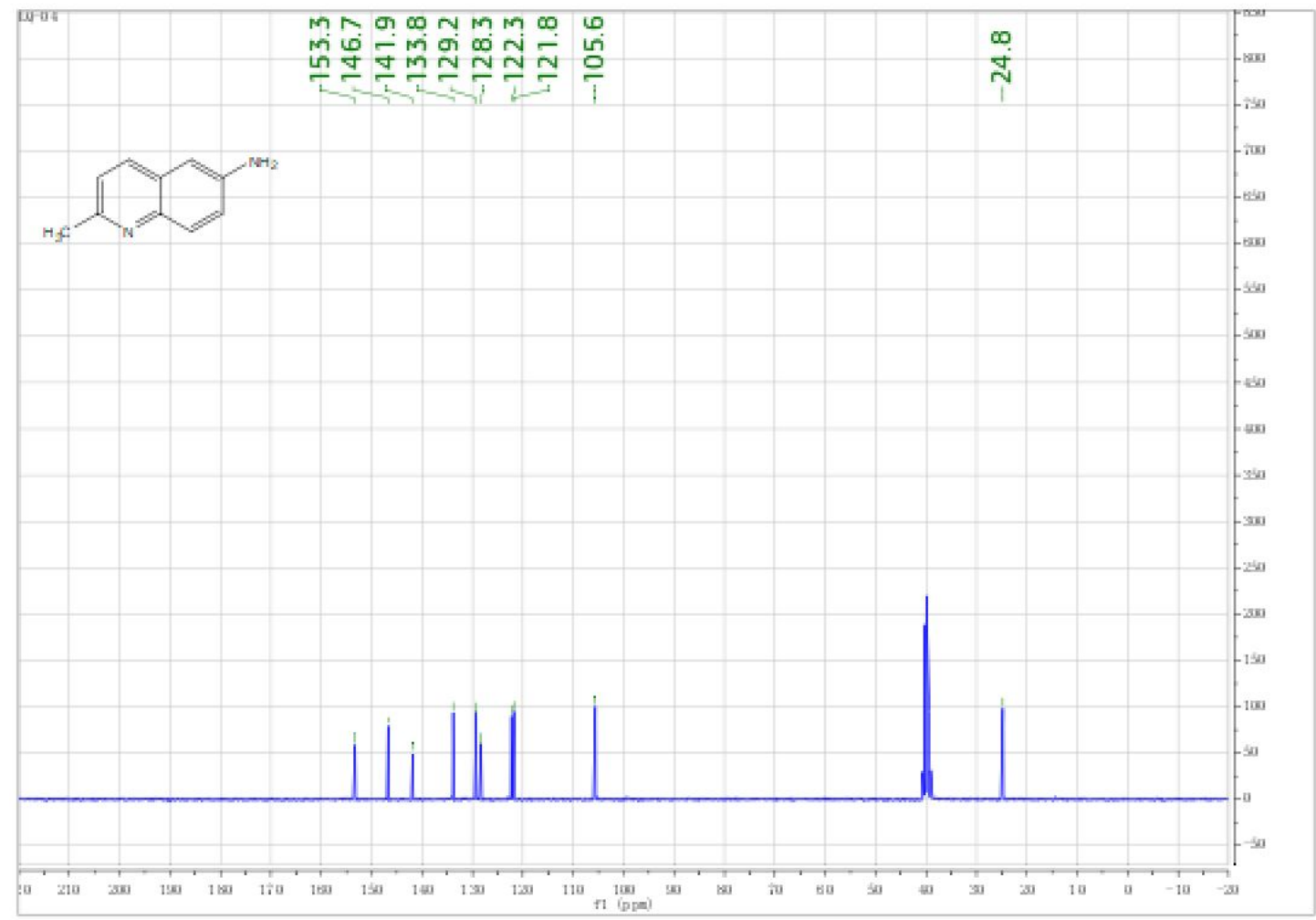

${ }^{1} \mathrm{H}$ NMR of intermediate compound 5. 


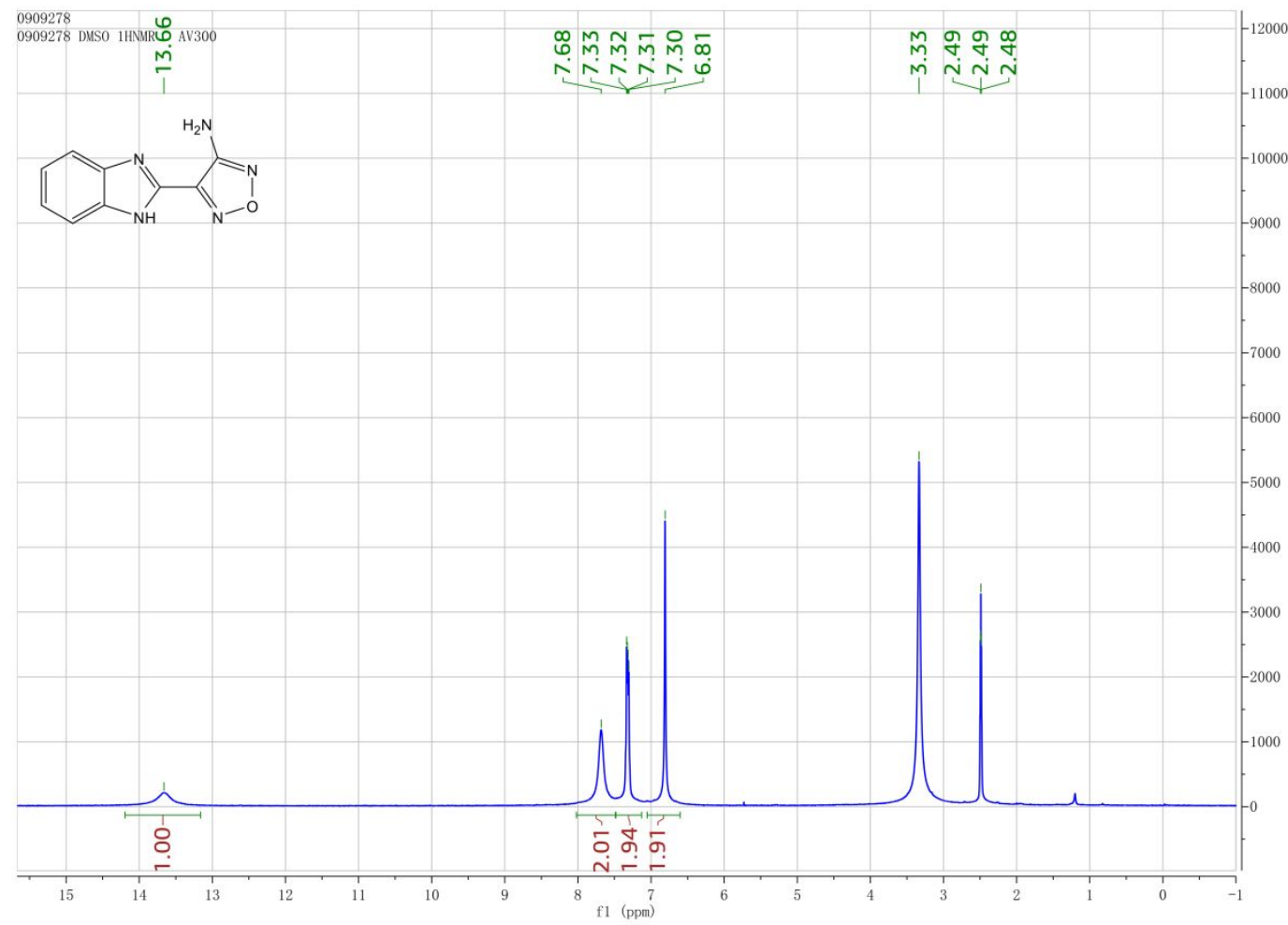

${ }^{13} \mathrm{C}$ NMR of intermediate compound $\mathbf{5}$.

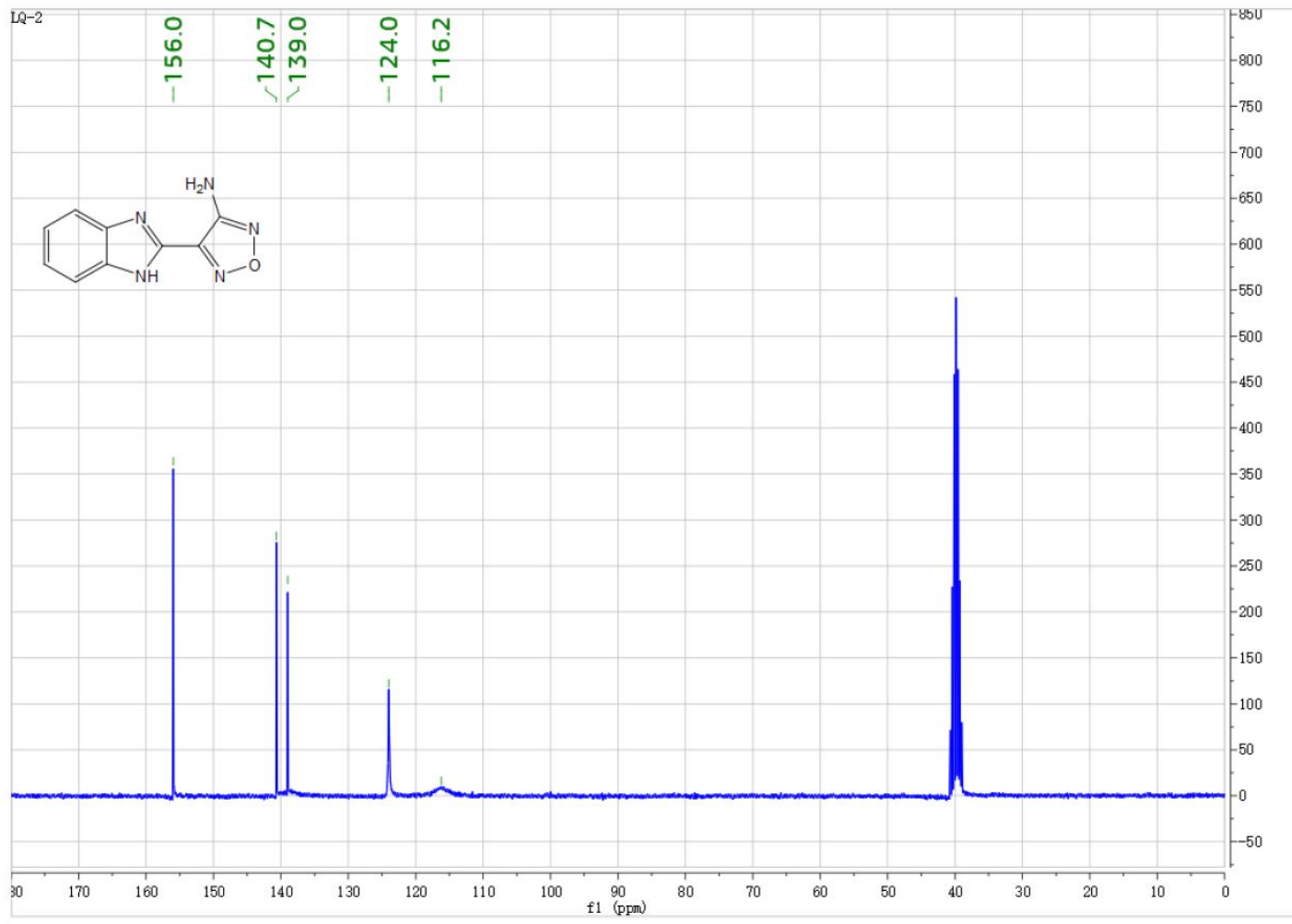

HPLC, ${ }^{1} \mathrm{H}$ NMR, ${ }^{13} \mathrm{C}$ NMR and HRMS of compound 8012-9656.

$\operatorname{HPLC}\left(\mathrm{t}_{\mathrm{R}}=5.1 \mathrm{~min}\right)$ : 


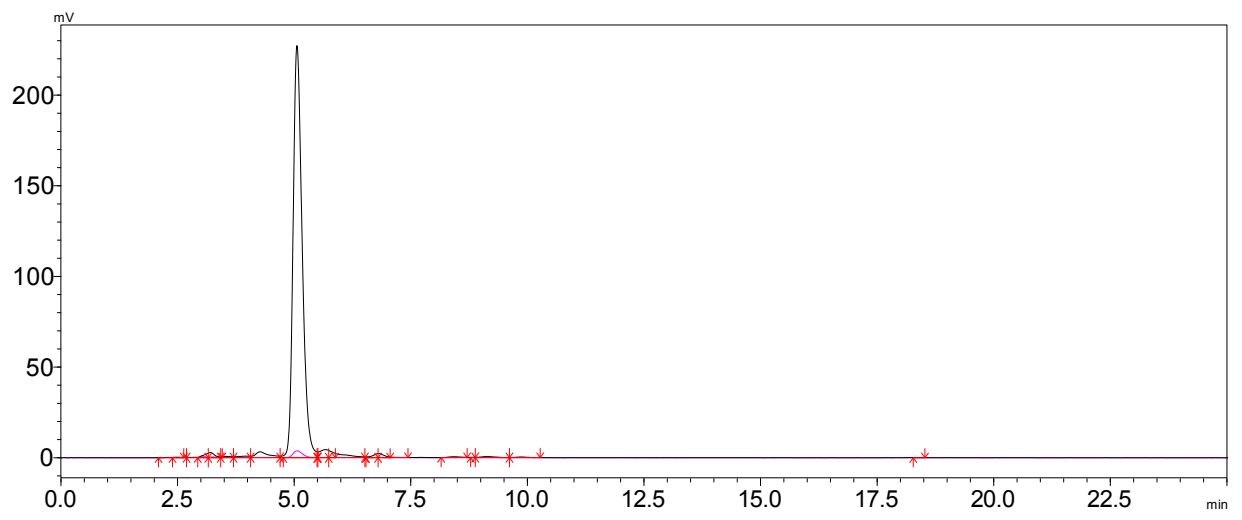

\section{${ }^{1} \mathrm{H}$ NMR}

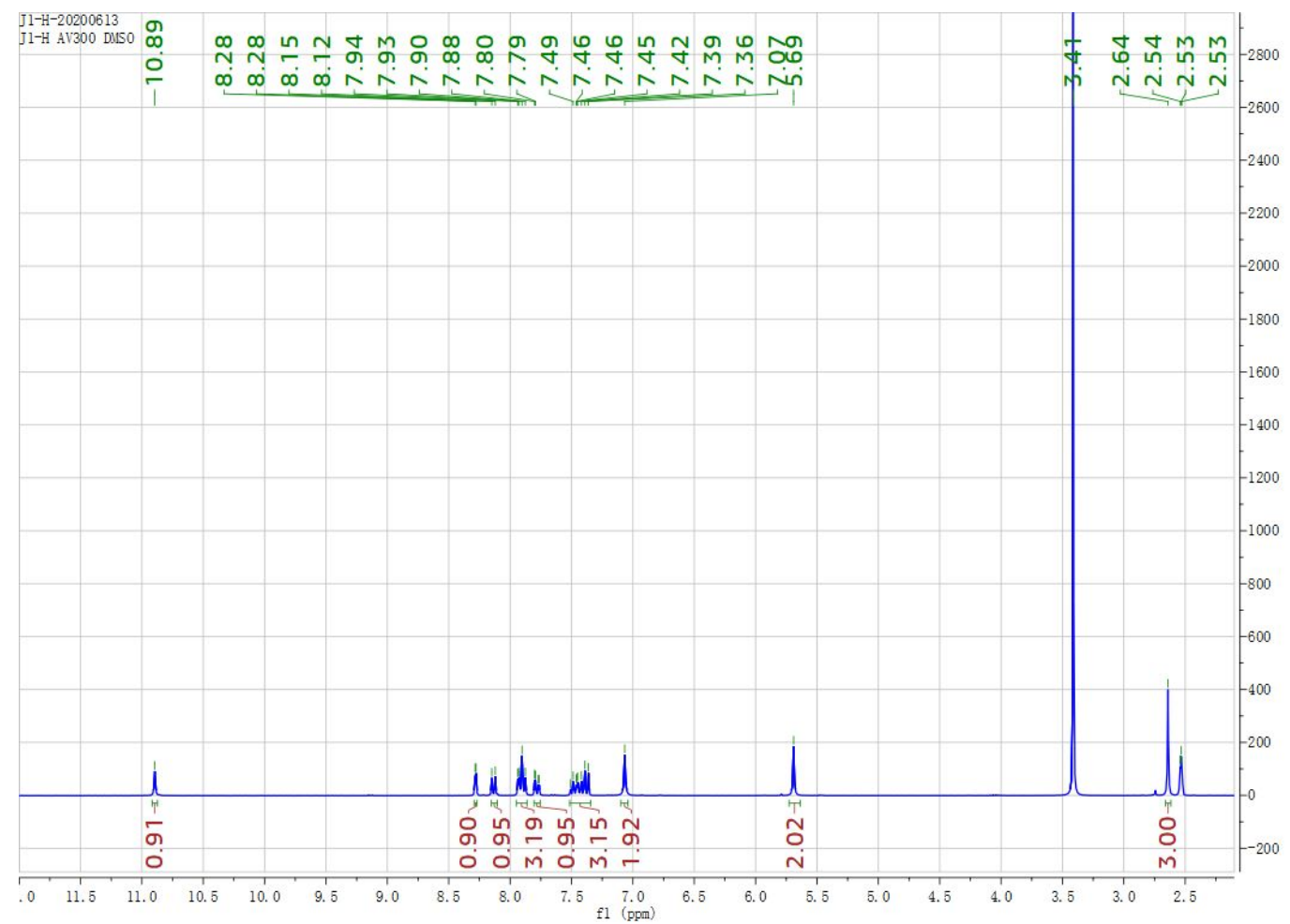

${ }^{13} \mathrm{C}$ NMR 


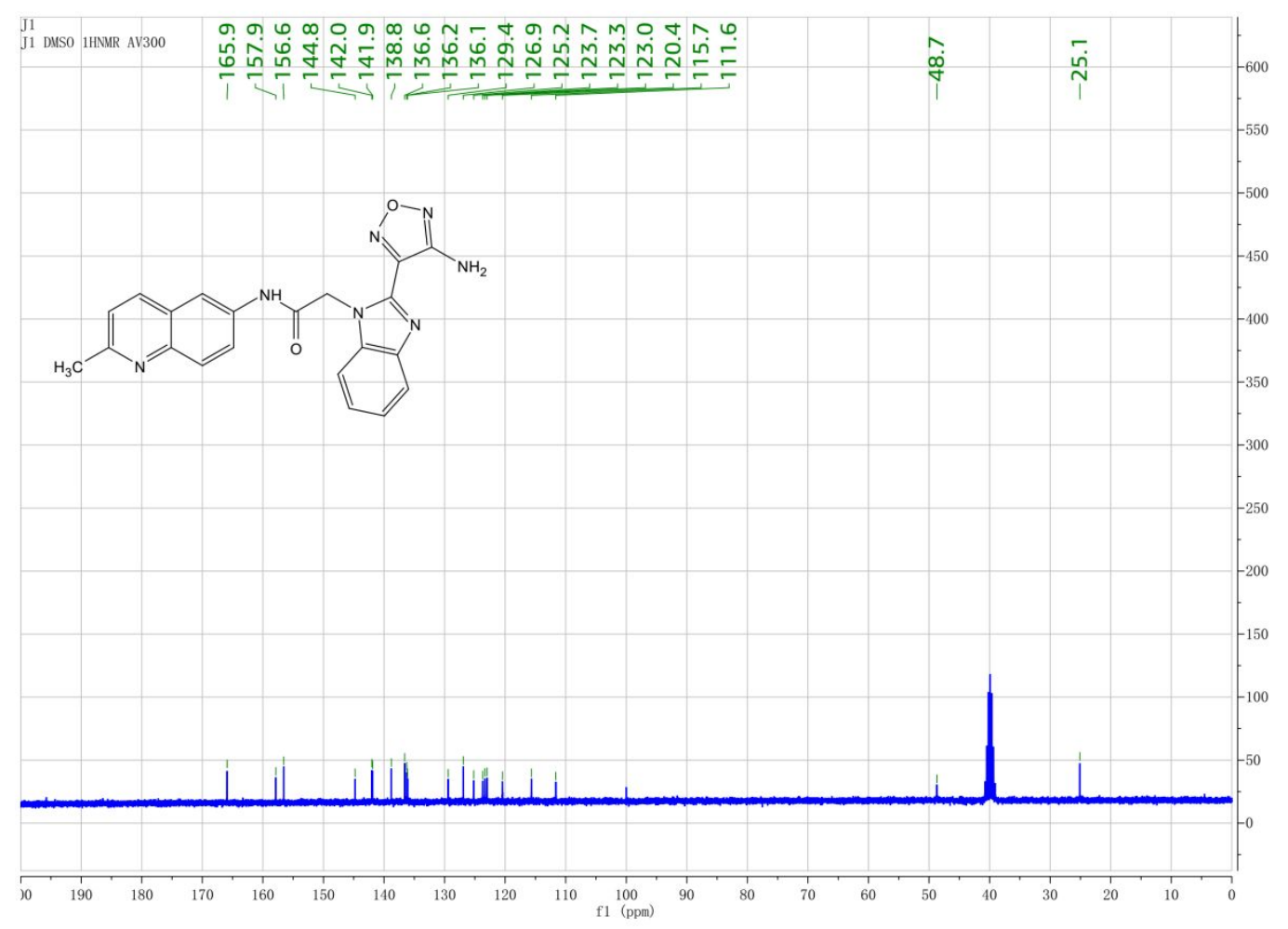

HRMS

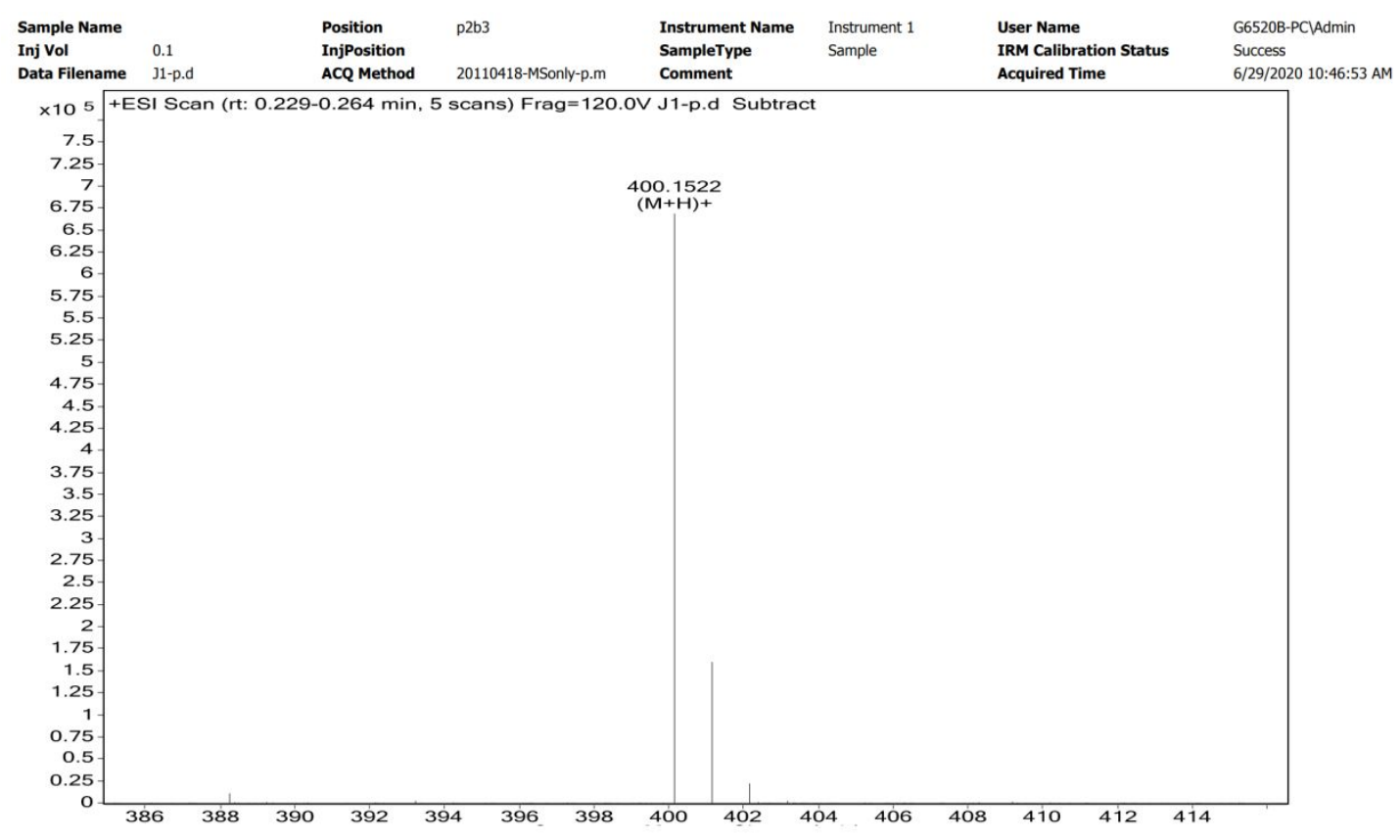

HPLC, ${ }^{1} \mathrm{H}$ NMR, ${ }^{13} \mathrm{C}$ NMR and HRMS of positive compound tacrine.

$\operatorname{HPLC}\left(\mathrm{t}_{\mathrm{R}}=2.6 \mathrm{~min}\right)$ 


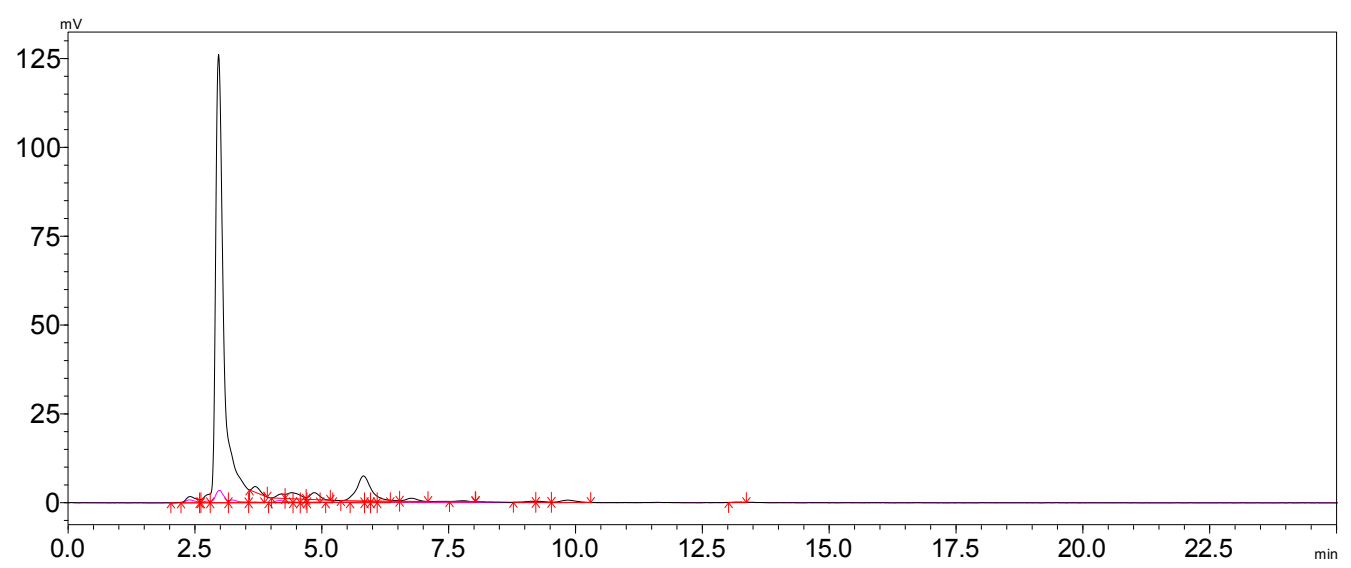

${ }^{1} \mathrm{H}$ NMR

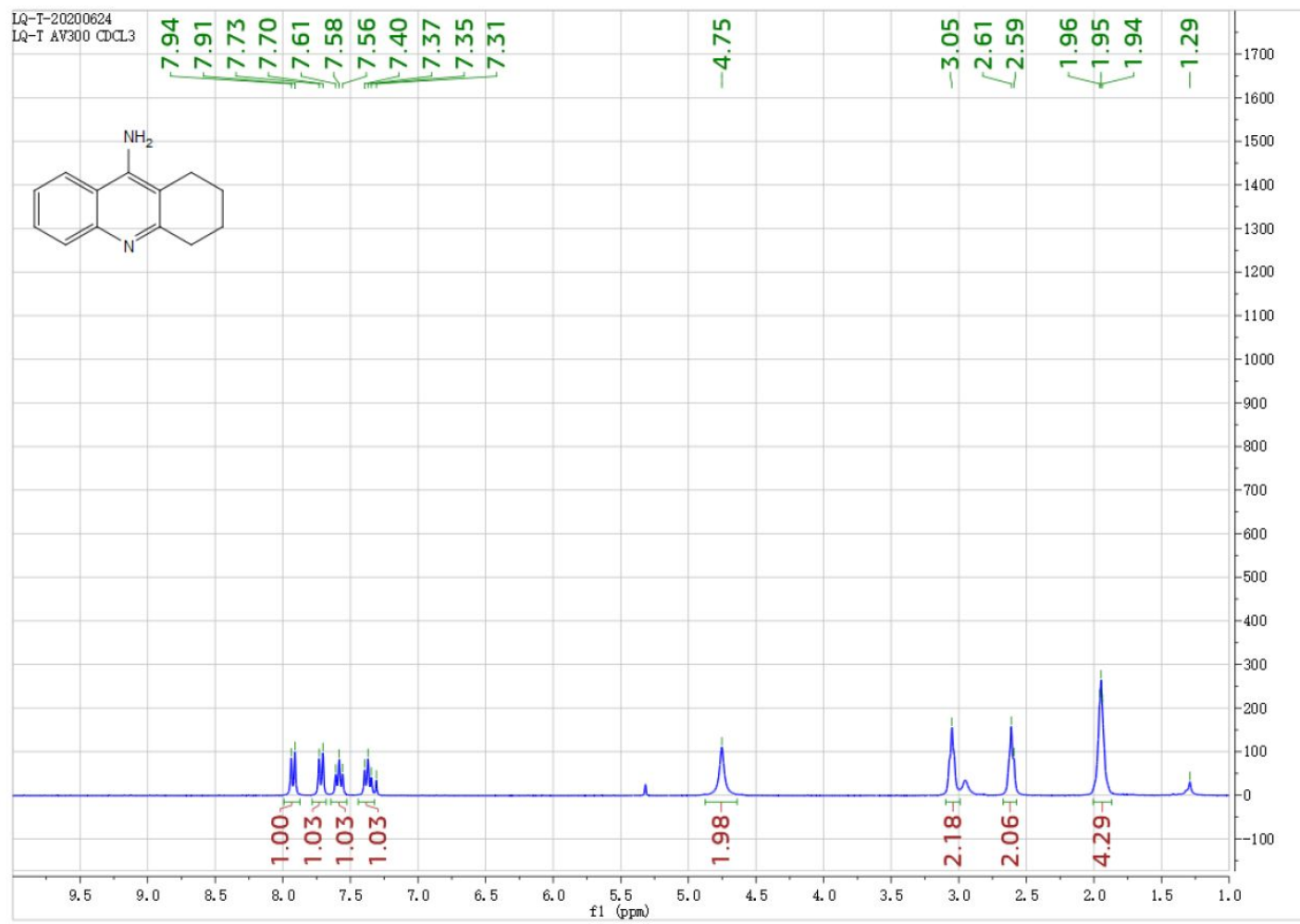

${ }^{13} \mathrm{C}$ NMR 


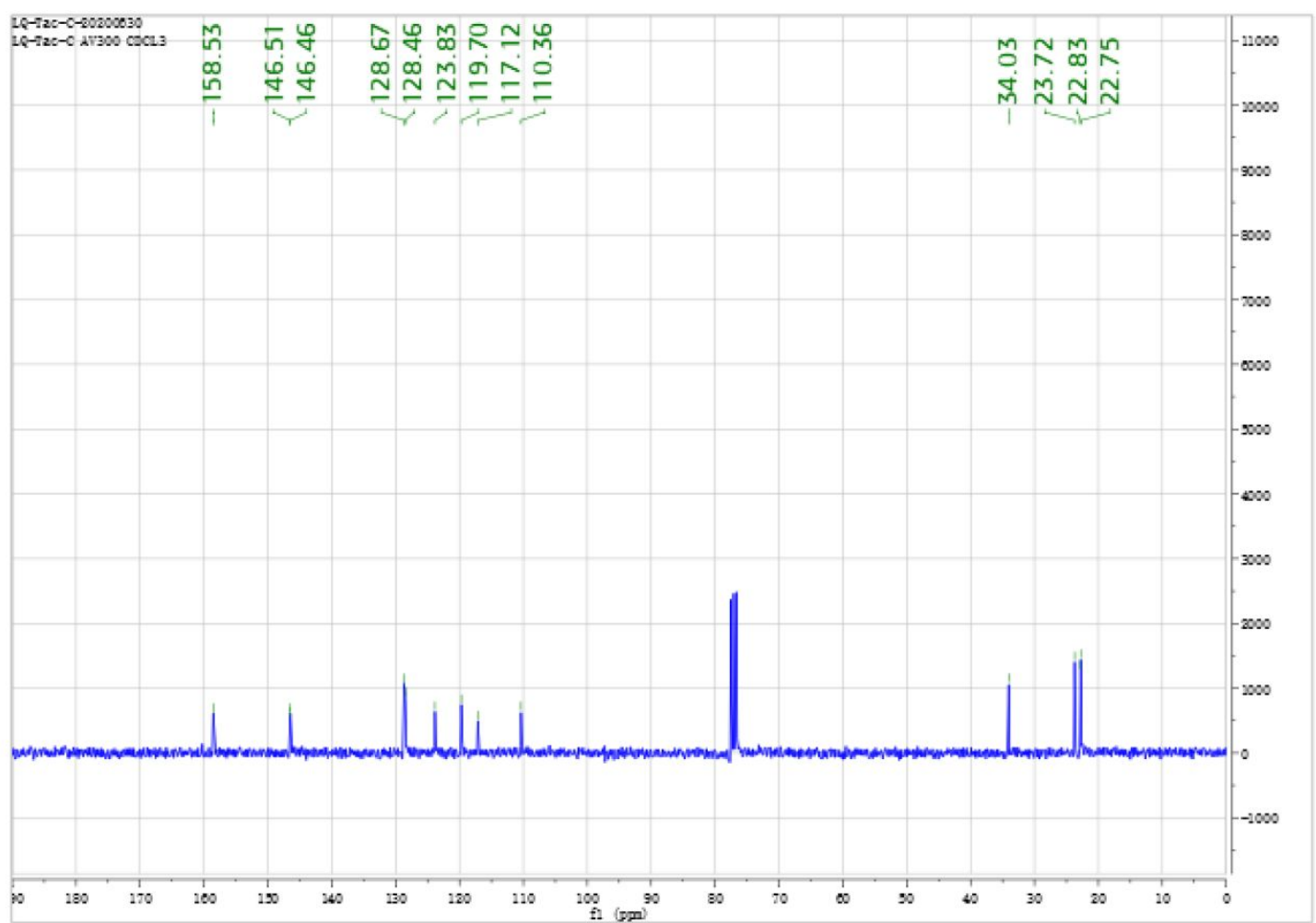

\section{HRMS}

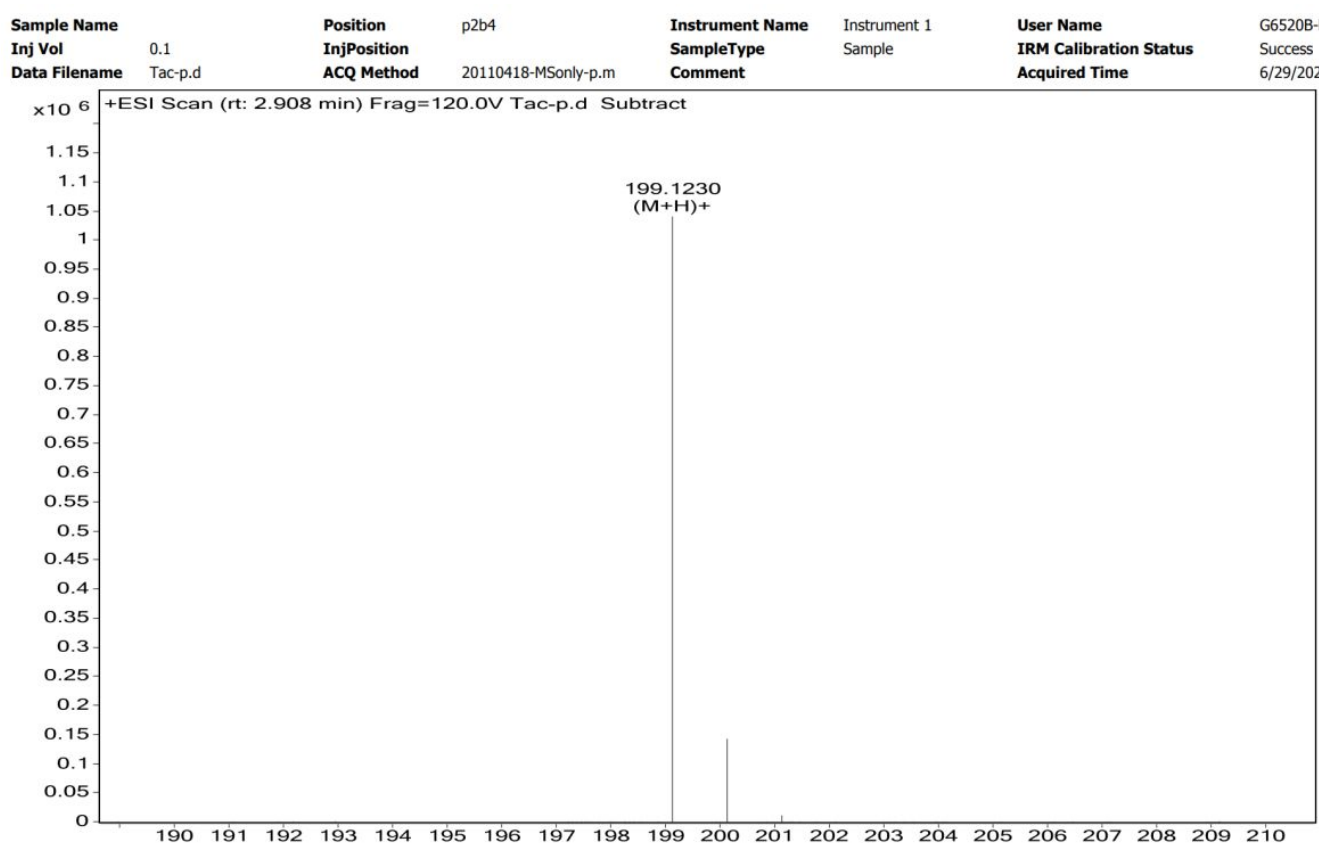

$\begin{array}{lllllllllllllllllllll}190 & 191 & 192 & 193 & 194 & 195 & 196 & 197 & 198 & 199 & 200 & 201 & 202 & 203 & 204 & 205 & 206 & 207 & 208 & 209 & 210\end{array}$ 
Table S2. Molecular Formula Strings of all compounds.

\begin{tabular}{|c|c|}
\hline Cpd. & Molecular Formula String \\
\hline 8015-2476 & $\mathrm{COC} 1=\mathrm{CC}(\mathrm{C} 2=\mathrm{CC}=\mathrm{CC}=\mathrm{C} 2 \mathrm{O} 3)=\mathrm{C} 3 \mathrm{C}=\mathrm{C} 1 \mathrm{NC}(\mathrm{C}(\mathrm{C}(\mathrm{C} 4=\mathrm{CC}=\mathrm{CC}=\mathrm{C} 4 \mathrm{~N} 5)$ \\
\hline 3434-1301 & $\mathrm{COC} 1=\mathrm{CC}=\mathrm{C}(\mathrm{C} 2=\mathrm{C}(\mathrm{NC} 3=\mathrm{CC}=\mathrm{C} 4 \mathrm{C}(\mathrm{C} 5=\mathrm{C}(\mathrm{O} 4) \mathrm{C}=\mathrm{CC}=\mathrm{C} 5)=\mathrm{C} 3) \mathrm{C} 6=\mathrm{C}(\mathrm{C} 2=\mathrm{O}) \mathrm{C}=\mathrm{CC}=\mathrm{C} 6) \mathrm{C}=\mathrm{C} 1$ \\
\hline V028-5347 & $\mathrm{COC} 1=\mathrm{C}(\mathrm{OC}) \mathrm{C}=\mathrm{C}(\mathrm{C} 2=\mathrm{CC}=\mathrm{CC}(\mathrm{N} 3 \mathrm{C}=\mathrm{NC}=\mathrm{C} 3 \mathrm{C}(\mathrm{N} 4 \mathrm{CC} 5=\mathrm{C}(\mathrm{C} 4) \mathrm{C}=\mathrm{CC}=\mathrm{C} 5)=\mathrm{O})=\mathrm{C} 2) \mathrm{C}=\mathrm{C} 1$ \\
\hline V011-7286 & $\mathrm{O}=\mathrm{C}(\mathrm{N}(\mathrm{CCC} 1=\mathrm{NC}(\mathrm{C} 2=\mathrm{CC}=\mathrm{C}(\mathrm{C}(\mathrm{F})(\mathrm{F}) \mathrm{F}) \mathrm{C}=\mathrm{C} 2)=\mathrm{NO} 1) \mathrm{CC} 3=\mathrm{CC}=\mathrm{CO} 3) \mathrm{C} 4=\mathrm{CC}=\mathrm{C}(\mathrm{C}(\mathrm{F})(\mathrm{F}) \mathrm{F}) \mathrm{C}=\mathrm{C} 4$ \\
\hline K630-0106 & $\mathrm{CC} 1=\mathrm{C}(\mathrm{NCC} 2=\mathrm{C}(\mathrm{O}) \mathrm{C}=\mathrm{CC}(\mathrm{CC} 3=\mathrm{CC}=\mathrm{CC}=\mathrm{C} 3)=\mathrm{C} 2) \mathrm{C}=\mathrm{C}(\mathrm{C} 4=\mathrm{NC} 5=\mathrm{CC}=\mathrm{CC}=\mathrm{C} 5 \mathrm{O} 4) \mathrm{C}=\mathrm{C} 1$ \\
\hline D101-0057 & $\mathrm{COC} 1=\mathrm{CC}=\mathrm{C}(\mathrm{C} 2=\mathrm{NOC}(\mathrm{CN}(\mathrm{C} 3 \mathrm{CCCCC} 3) \mathrm{C}(\mathrm{C} 4=\mathrm{CC} 5=\mathrm{C}(\mathrm{OCO} 5) \mathrm{C}=\mathrm{C} 4)=\mathrm{O})=\mathrm{N} 2) \mathrm{C}=\mathrm{C} 1$ \\
\hline M769-0012 & $\mathrm{CC}(\mathrm{C})(\mathrm{C}) \mathrm{C} 1=\mathrm{CC}=\mathrm{C}(\mathrm{C}(\mathrm{N}(\mathrm{CCC} 2=\mathrm{NOC}(\mathrm{C} 3=\mathrm{CC} 4=\mathrm{CC}=\mathrm{CC}=\mathrm{C} 4 \mathrm{~N} 3)=\mathrm{N} 2) \mathrm{C})=\mathrm{O}) \mathrm{C}=\mathrm{C} 1$ \\
\hline M769-0033 & $\mathrm{CN}(\mathrm{C}) \mathrm{C} 1=\mathrm{CC}=\mathrm{C}(\mathrm{C}(\mathrm{N}(\mathrm{CCC} 2=\mathrm{NOC}(\mathrm{C} 3=\mathrm{CC} 4=\mathrm{CC}=\mathrm{CC}=\mathrm{C} 4 \mathrm{~N} 3)=\mathrm{N} 2) \mathrm{C})=\mathrm{O}) \mathrm{C}=\mathrm{C} 1$ \\
\hline V009-8861 & $\mathrm{CC} 1=\mathrm{CC}=\mathrm{C}(\mathrm{OCC} 2=\mathrm{NC}(\mathrm{CN}(\mathrm{CC}(\mathrm{C}) \mathrm{C}) \mathrm{C}(\mathrm{C} 3=\mathrm{CC} 4=\mathrm{C}(\mathrm{OCO} 4) \mathrm{C}=\mathrm{C} 3)=\mathrm{O})=\mathrm{CS} 2) \mathrm{C}=\mathrm{C} 1$ \\
\hline P702-0211 & $\mathrm{CC} 1=\mathrm{CC}=\mathrm{C} 2 \mathrm{C}(\mathrm{NC} 3=\mathrm{CC}=\mathrm{C}(\mathrm{OCCO} 4) \mathrm{C} 4=\mathrm{C} 3)=\mathrm{C}(\mathrm{C} 5=\mathrm{NC}(\mathrm{CC})=\mathrm{NO} 5) \mathrm{C}=\mathrm{NC} 2=\mathrm{N} 1$ \\
\hline V007-7549 & $\mathrm{CC} 1=\mathrm{CC}=\mathrm{C}(\mathrm{OCC} 2=\mathrm{NC}(\mathrm{CN}(\mathrm{CCOC}) \mathrm{C}(\mathrm{C} 3=\mathrm{CC}=\mathrm{C}(\mathrm{OC}) \mathrm{C}=\mathrm{C} 3)=\mathrm{O})=\mathrm{CS} 2) \mathrm{C}=\mathrm{C} 1$ \\
\hline V013-8206 & $\mathrm{CC} 1=\mathrm{CC}=\mathrm{C}(\mathrm{OCC} 2=\mathrm{NC}(\mathrm{CN}(\mathrm{CCC}) \mathrm{C}(\mathrm{C} 3=\mathrm{CC} 4=\mathrm{C}(\mathrm{OCO} 4) \mathrm{C}=\mathrm{C} 3)=\mathrm{O})=\mathrm{CS} 2) \mathrm{C}=\mathrm{C} 1$ \\
\hline $8012-9656$ & $\mathrm{CC} 1=\mathrm{NC}(\mathrm{C}=\mathrm{CC}(\mathrm{NC}(\mathrm{CN}(\mathrm{C}(\mathrm{C} 2=\mathrm{NON}=\mathrm{C} 2 \mathrm{~N})=\mathrm{N} 3) \mathrm{C} 4=\mathrm{C} 3 \mathrm{C}=\mathrm{CC}=\mathrm{C} 4)=\mathrm{O})=\mathrm{C} 5)=\mathrm{C} 5 \mathrm{C}=\mathrm{C} 1$ \\
\hline Tacrine & $\mathrm{NC} 1=\mathrm{C} 2 \mathrm{C}(\mathrm{CCCC} 2)=\mathrm{NC} 3=\mathrm{CC}=\mathrm{CC}=\mathrm{C} 31$ \\
\hline
\end{tabular}

